\title{
Cuidados y diversidad cultural: un estudio de caso en un centro de Atención Primaria en Murcia
}

\author{
Nursing care and cultural diversity: a case study in \\ a Primary Health Care Center in Murcia
}

\section{Cuidados e diversidade cultural: um estudo de caso do Unidade de Cuidados de Saúde Primários em Murcia}

\author{
Carmen Martínez Cano ${ }^{1}$, Salvador Cayuela Sánchez ${ }^{2}$ \\ ${ }^{1}$ Residente de Enfermería Familiar y Comunitaria (Unidad Docente Centro. Comunidad de Madrid) \\ ${ }^{2}$ Doctor en Filosofía (Universidad de Murcia). Doctor en Antropología Social (Universidad Rovira i Virgili). \\ Prof. Ayte. Dr. Facultad de Medicina de Albacete (UCLM). \\ Cómo citar este artículo en edición digital: Martínez Cano, C., \& Cayuela Sánchez, S. (2018). Cuidados y diversidad cultural: un \\ estudio de caso en un centro de Atención Primaria en Murcia. Cultura de los Cuidados (Edición digital), 22(50). \\ Recuperado de http://dx.doi.org/10.14198/cuid.2018.50.12 \\ Correspondencia: Facultad de Enfermería (Universidad de Murcia). \\ Correo electrónico: carmen.martinez20@um.es \\ Recibido: 17/06/2017; Aceptado: 23/10/2017
}

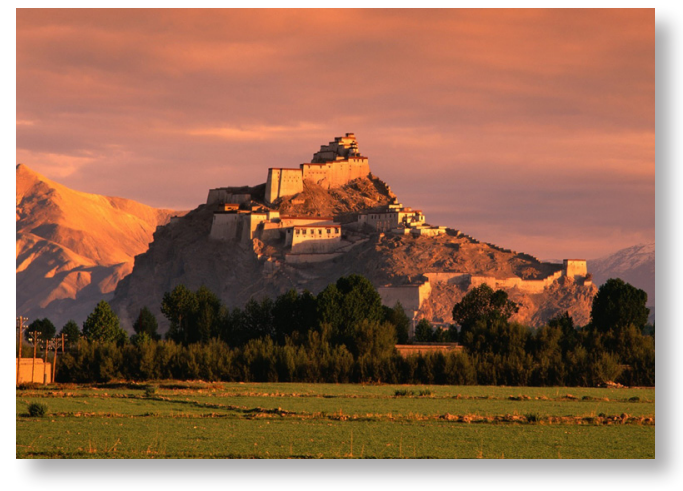

ABSTRACT

Objetive: the purpose of this study is to analyze and interpret how a group of nurses working at a Primary Health Care Center perceives cultural diversity. This involves describing their relationship with migrants, taking into account the attitude they have and the strategies they employ when attending these patients.

Methodology: qualitative design through semi-structured interviews developed in a Pri- mary Health Care Center in Murcia (Spain). Analysis was carried out through coding, saturation and triangulation of the interviews, with the support of the data analysis software MaxQda

Results: this group of nurses associate cultural diversity with economic migrants, and label them in different ways. Their attitude in this respect strives to give equal health care to every patient. However, the language and cultural barrier they face on a daily basis makes them disagree on the clinical practice, so they suggest strategies to overcome this issue.

Final thoughts: the presence of patients from diverse cultures in health care and the importance of providing culturally consistent care are obvious facts. Language and cultural barriers, as well as social prejudices and stereotypes, represent a barrier to establish a therapeutic relationship and practice Health Education. 
Key words: Nurse-Patient Relations, Cultural Diversity, Migration, Primary Health Care, Qualitative Study.

\section{RESUMEN}

Objetivo: analizar e interpretar la percepción que un grupo de enfermeras de Atención Primaria tiene acerca de la diversidad cultural y describir sus actitudes y estrategias en la atención a la salud.

Método: estudio de enfoque cualitativo mediante entrevistas semiestructuradas desarrolladas en un Centro de Atención Primaria de la Región de Murcia (España). Análisis a través de codificación, saturación y triangulación de las entrevistas, con el apoyo del programa informático de codificación MaxQda ${ }^{\oplus}$.

Resultados: este grupo de enfermeras asocia la diversidad cultural con el perfil de inmigrante económico, al cual asignan diversas etiquetas. Muestran una actitud que persigue la equidad, pero tienen desencuentros en la práctica clínica relacionados con la barrera lingüística y cultural, para los que sugieren estrategias.

Consideraciones finales: la presencia de pacientes culturalmente diversos en el ámbito de la salud es evidente y también la importancia de proporcionar cuidados culturalmente congruentes. Las dificultades relacionadas con la barrera lingüística y cultural, así como los prejuicios y estereotipos, representan para este grupo de enfermeras un obstáculo para establecer la relación teraéutica y la práctica de EpS.

Palabras clave: Relación Enfermera-Paciente, Diversidad Cultural, Migración Internacional, Atención Primaria de Salud, Estudio Cualitativo.

\section{RESUMO}

Objetivo: análise e interpretação a perceção que um grupo de enfermeiras de Cuidados de Saúde Primários tem acerca da diversidade cultural e descrever como é a sua relação com a população imigrante, através das suas atitudes e estratégias em cuidados de saúde.

Metodologia: abordagem cualitativa por meio de entrevistas semiestruturadas numa Unidade de Cuidados de Saúde Primários da Região de Múrcia (Espanha). Análise qualitativa através de codificação, saturação e triangulação das entrevistas transcritas, com apoio de um programa informático de codificação MaxQda ${ }^{\oplus}$.

Resultados: este grupo de enfermeiras associa à diversidade cultural com o perfil de imigrante económico, ao qual são atribuídos diferentes peculiaridades. Neste sentido, observa-se uma atitude que persegue a igualdade na assistência sanitária, mas tem desencontros com a prática clínica, relacionados com a recorrente barreira linguista e cultural.

Considerações finais: a presença de pacientes culturalmente diversificada no campo da saúde é evidente e também a importância para prestar cuidados culturalmente congruentes. as dificuldades relacionadas com as barreiras linguísticas e culturais, bem como preconceitos e estereótipos, representa um obstáculo para este grupo este grupo de enfermeiras para estabelecer na relação teraéutica e na EpS.

Palavras-chave: Relação Enfermeira-Utente, Diversidade Cultural, Migração Internacional, Cuidados de Saúde Primária, Estudo Qualitativo.

\section{INTRODUCCIÓN}

Las sociedades occidentales están sujetas a un permanente cambio en su composición cultural. La variabilidad de su realidad sociocultural ha sido significativa en los últimos 
años debido al aumento de extranjeros -fenómeno que tiene repercusiones en el ámbito de la salud, empleo, vivienda, educación y otros pilares que sostienen el llamado Estado de bienestar-. En materia de atención a la salud, los servicios sanitarios públicos han de atender las necesidades que genera la diversidad cultural no solo entendida como la variabilidad en cuanto a nacionalidad, religión, edad, género, orientación sexual, capacidad y discapacidad, estatus socioeconómico, nivel educativo, etc., sino también como la diversidad de valores, creencias, visión del mundo, de la vida, de la muerte y del proceso salud-enfermedad (de cómo se enferma, cómo se sana, quién puede sanar, etc.) (Leno, 2006). Los profesionales de Enfermería, ante el aumento de los flujos migratorios y en su contacto directo hacia el nuevo perfil de usuario, han incrementado su interés y se han sumado a estudios antropológicos con el fin de prestar cuidados culturalmente congruentes (Lillo y Casabona, 2006). Países como Estados Unidos, Reino Unido, Canadá y Australia, ya contemplan en el cuerpo de conocimientos de sus profesionales sanitarios habilidades relacionadas con la competencia cultural definida por la Academia Americana de Enfermería (ANA) como: “Tener conocimiento, actitudes y habilidades sobre grupos culturales diversos que permite al profesional de la salud proporcionar cuidado cultural de acuerdo a las necesidades manifestadas por el paciente. Es un proceso continuo que involucra aceptación y el respecto de las diferencias y no permite que las creencias personales propias tengan una excesiva influencia en aquellos que tienen una visión del mundo diferente a la propia” (Gentil, 2010).

En 1946, la OMS señala que gozar del grado máximo de salud que se pueda lograr es uno de los derechos fundamentales de todo ser humano sin distinción de raza, religión, ideología política o condición económica o social (Dept. of Ethics, Trade, Human Rights and Health Law. OMS, 2005). En la década de los 80 , esta institución reconoce la cultura y la diversidad cultural como aspectos a tener en cuenta en los cuidados para la salud afirmando que "un elemento crucial del derecho a la salud es que todos los establecimientos, bienes y servicios de salud deben ser culturalmente apropiados" (Serra et al, 2013).

Cuando se trata de extranjeros o inmigrantes se adquiere mayor conciencia de las diferentes culturas, lo que con frecuencia se acompaña de una serie de actitudes, estereotipos y prejuicios adoptados por el profesional sanitario, que suponen tanto una barrera en la utilización de los servicios sanitarios por parte del inmigrante, como un reto y/o una dificultad en la prestación de cuidados de Enfermería (Plaza, 2008; Moreno, 2008; Rico, 2009).

Entre las particularidades del diagnóstico de salud de la población inmigrante, destacan la dificultad en el acceso y la utilización de Servicios Sanitarios, relacionada con factores propios del inmigrante -diferencia de idioma, problemas de comunicación, miedo, desconfianza, creencias y valores culturales, uso de la medicina tradicional y natural y/o conceptos de salud, cuidados y enfermedad-, del profesional de la salud -percepción de abuso, sobrecarga asistencial, desconocimiento de la cultura, estereotipos y estigmatización- y del Sistema de Salud -procesos burocráticos, requisitos administrativos, gestión de consultas, horarios, falta de material adaptado culturalmente, etc.-. De esta situación se deriva que, pese a que las necesidades básicas sean similares para toda la población -salud, empleo y vivienda-, el colectivo de inmigrantes presenta mayor probabilidad de sufrir enfer- 
medades crónicas y enfermedades psicosomáticas derivadas del proceso de adaptación al nuevo medio. Además, presenta limitaciones en el manejo de las mismas, lo que ha llevado a considerar la condición de inmigrado como un factor de riesgo para la salud (Belintxon y Lopez-Dicastillo, 2010).

Los movimientos migratorios, tanto dentro de un país como entre países, han dado lugar a un paisaje multicultural en la población nacional y global. En los últimos 10 años, España ha recibido 5 millones de nuevos habitantes, los cuales llegaron a representar el 12\% de la población en 2010 (Ministerio de Trabajo e Inmigración, 2011). Con la recesión económica, se han incrementado los flujos de retorno. No obstante, no se puede ignorar el peso del remplazo generacional que acompaña a esta población caracterizada por ser joven, en edad laboral y con alta tasa de fecundidad. En este punto, la Región de Murcia se sitúa como una de las comunidades autónomas con la ratio más alta de extranjeros sobre la población total en el conjunto de España. El porcentaje de extranjeros residentes en la región alcanzaba el $16,4 \%$ sobre el total de la población en 2012. A pesar de la situación económica actual y al descenso del saldo migratorio, la Región de Murcia sigue siendo receptora de población extranjera (Consejería de Economía y Hacienda, 2012).

El Sistema Sanitario y su cuerpo de profesionales están sujetos a un modelo médico dirigido a lo psicosomático -esto es, al proceso patológico frente al enfermo y su subjetividad-. Este enfoque forma parte de la cultura de salud occidental cuyo eje es la Medicina Científica Moderna o Biomedicina (Perdiguero y Comelles, 2000). Sin embargo, ante las necesidades de una sociedad abierta y culturalmente diversa, y con el objetivo de proporcionar cuidados centrados en el paciente, se hace necesario el estudio de la dimensión cultural del cuidado. De hecho, el interés por la prestación de cuidados de Enfermería adaptados a la cultura ya fue reclamado por Madeleine Leininger, fundadora de la Enfermería Transcultural, en la década de 1960. Esta enfermera y antropóloga resalta la importancia de estudiar a las personas, a través de metodología cualitativa, desde el punto de vista de sus conocimientos y experiencias locales o emic, para contrastarlo con los factores etic, o prácticas y convicciones de los profesionales de Enfermería. Se persigue así generar una aproximación holística al estudio de las conductas culturales en diversos contextos ambientales (Leno, 2006).

\section{OBJETIVOS}

Tomando todo esto como punto de partida, en este trabajo pretendemos indagar acerca de cómo convive con esta realidad un grupo de profesionales de Enfermería que actualmente realiza su labor asistencial en un Centro de Atención Primaria. Perseguimos por tanto interpretar la percepción que los profesionales de Enfermería tienen acerca de la diversidad cultural, para lo cual trataremos de describir la actitud y las estrategias que nuestras entrevistadas sostienen en la asistencia a una población multicultural. Finalmente, nos proponemos identificar la necesidad de una intervención formativa para la mejora de la atención a la diversidad cultural dirigida a los profesionales de Enfermería.

\section{METODOLOGÍA}

En un inicio, los estudios sobre inmigración y salud se centraron principalmente en la enfermedad, lo que ha señalado que no enferman más que los autóctonos y desestima el impacto de las patologías importadas pese al 
discurso mediático (Fuertes et al, 2010). Sin embargo, como ya mencionábamos, existe un estado de vulnerabilidad por la condición de inmigrado. Puesto que realizar el diagnóstico de salud de una población multicultural es también explorar a los profesionales por los que es asistida, son numerosos los estudios encaminados a estudiar el cuidado del "otro". El abordaje de esta cuestión, y en concreto de la relación paciente-enfermera, se ha enfocado mayormente hacia la percepción que el profesional tiene del inmigrante, bien a través de cuestionarios desde la metodología cuantitativa, o bien a través técnicas como la entrevista en profundidad, la observación participante o los grupos de discusión, desde una vertiente cualitativa.

A través de este estudio, en su acercamiento a la realidad de la relación pacienteenfermera, pretendemos contribuir al cuerpo de conocimientos enfermero desde una perspectiva antropológica. Si bien, como disciplina profesional holística, la Enfermería española todavía está lejos de crear y unificar estrategias para el cuidado culturalmente adaptado.

Partiendo de estos análisis, y para responder a los objetivos propuestos, utilizaremos aquí una metodología cualitativa desde un enfoque fenomenológico, con la finalidad de describir el significado que para el profesional enfermero tiene la población multicultural y cómo sus experiencias y percepciones condicionan la construcción de la relación con ellos. La comprensión de la percepción humana requiere que la persona, integrada en su entorno, interprete la acción o experiencia para el investigador, quien después debe interpretar la explicación dada por la persona (Burns y Grove, 2012). Las respuestas de los informantes poseen valor porque, aunque en cierto modo son fi de error, en otro son hechos sociales sig-

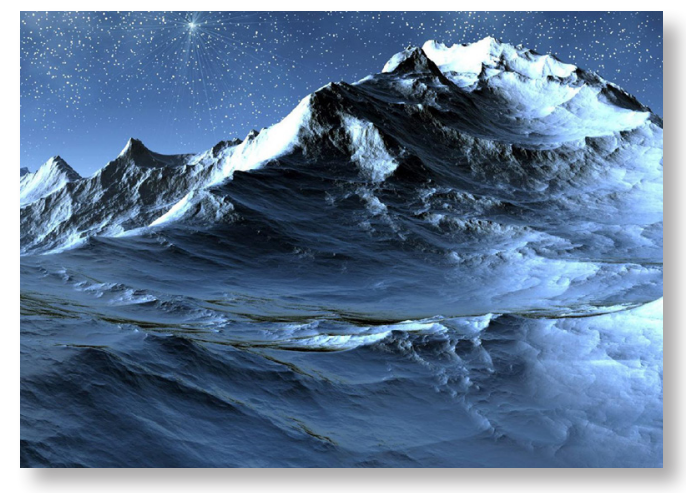

nificativos y gérmenes de conocimiento. En este sentido, en efecto, la información verbal sobre la acción social es acción ella misma (Jociles, 1999).

Tomando en consideración los precedentes anteriormente expuestos y asumiendo estas precauciones metodológicas y analíticas, decidimos desarrollar nuestro análisis cualitativo en el ámbito de Atención Primaria, donde es posible un mayor seguimiento y más amplia interacción con los hábitos y estilos de vida del paciente. Ocho de las once enfermeras que constituyen la plantilla del centro, entre las cuales se incluyen la matrona y la enfermera pediátrica, accedieron a participar en el estudio. La recogida de datos en el trabajo de campo se llevó a cabo mediante la realización de entrevistas semiestructuradas, iniciadas con una pregunta abierta - ¿Cuál es tu percepción acerca de la diversidad cultural como enfermera?"-, precedidas claro de la pertinente información acerca de los objetivos del estudio y del requerido consentimiento informado. A continuación -y dependiendo siempre de la actitud y facilidad de palabra de la informante-, se formularon preguntas del tipo “ ¿Cómo describirías tus estrategias en la consulta ante esta situación?”, o “CCómo definirías tu actitud ante una población multicultural?”. Finalmente, y para cerrar la entrevista, se preguntaba acerca de la necesidad de una formación destinada 
a mejorar el aspecto de la asistencia a la población culturalmente diversa en Enfermería. A continuación, las entrevistas registradas en audio fueron transcritas y codificadas mediante la utilización del programa informático de análisis cualitativo MaxQda ${ }^{\circledR}$.

\section{RESULTADOS}

\section{Percepciones de la diversidad cultural}

A continuación, exponemos la visión general que las profesionales entrevistadas reflejan acerca de la diversidad cultural, la cual señala que la presencia de población multicultural es un fenómeno reconocido y aceptado con naturalidad entre las enfermeras, que se justifica por la globalización y la configuración de las conocidas como "sociedades abiertas". Así lo exponen algunas de nuestras entrevistadas:

"El tema de la diversidad cultural es un tema que ahora mismo vivimos con ello cada día. Parece que cada día tiende más el mundo a abrir barreras y en general yo veo que se acepta bastante bien el tema de que ahora no estemos cada uno nada más en nuestro país sino que haya personas de todos los lugares del mundo" (Enfermera 1)

"[...] hoy día en el mundo no hay distancias. Entonces precisamente hay que trabajar eso mucho. [...], no hay fronteras o no debe de haberlas. Entonces es algo que nos implica mucho la ampliación de conocimientos de otras culturas" (Enfermera 3)

Es destacable que, desde el inicio de las entrevistas, la gran mayoría asocia la diversidad cultural con el perfil de un paciente extranjero o inmigrante, y en concreto del inmigrante económico entendido como "aquella persona extranjera que entra dentro del territorio nacional, con el propósito de mejorar o cambiar una situación económica y con el fin de mejo- rar su nivel de vida" (Rico, 2009). El perfil tipo identificado suele corresponderse con aquel que genera mayor conciencia de diferencia cultural y lingüística -como son el paciente de origen asiático o el paciente musulmán-. De ahí que la principal barrera señalada en la labor asistencial sea el idioma, y en segundo lugar el estilo de vida dentro del cual destacan los hábitos alimenticios, como manifiestan algunas de las enfermeras interrogadas:

"En cuestión de la diversidad cultural aquí lo que más se ve, que son inmigrantes y que claro, que precisamente no vienen con niveles culturales muy altos, sino con niveles culturales medio-bajo [...] Pero te genera un problema importante a la hora de entenderte con ellos porque hay un problema de... por supuesto lingüístico [...]" (Enfermera 4)

"[...] sí que te puedo decir las tres culturas que tengo ahora mismo. Tengo a los árabes, a los chinos y europeos del Este. Los tres son bastante cumplidores. [...] no se niegan a poner vacunas pero sí que es verdad que la alimentación ahí tengo que hacer mucho hincapié" (Enfermera 8)

Pese a que el guion de la entrevista no estaba orientado a extraer aspectos problemáticos, son varios los discursos que avanzan en esa dirección, sucumbiendo a generalizaciones $\mathrm{y}$ atribuyendo una serie de cualidades relacionadas con un bajo nivel socioeconómico, con conflictividad, enfermedad e incluso experiencias que se restringen a destacar lo exótico. No obstante, con el propio desarrollo de la entrevista, a menudo desmienten deslizarse hacia estas posturas, lo cual sugiere la presencia de prejuicios de este tipo entre los profesionales de Enfermería, y la propia conciencia crítica de los mismos: 
"Estamos en un centro muy privilegiado precisamente, [...] este tema se ve poco, se ve muy poco. Pero hay zonas de la periferia que son muy conflictivas, donde hay un número altísimo de personas extranjeras y desde luego no se trabaja igual que aquí. [...] Tampoco cumplen las normas, quiero decir, a una persona de aquí tú le dices [...] que tiene que llevar unas normas, que... Y los extranjeros no. Los extranjeros quieren saltárselo todo a la torera. Ellos están aquí y son los que primeros necesitan la asistencia [...]. Y te generan ese tipo de problemas muchas veces $y$ otras no. Muchas veces, sí se creen con muchos más derechos de los que realmente tienen" (Enfermera 4)

“Crees que el hecho de ser inmigrante puede tener alguna repercusión sobre su salud?" (Entrevistadora)

"No tiene por qué. Depende de los recursos. Obviamente, si es un inmigrante que tiene mucha falta de recursos pues sí que puede ser que esté malnutrido, que tenga más heridas, a lo mejor que otra persona. Que podría ser, pero yo no me lo he encontrado. [...] No me he encontrado ningunas grandes diferencias de que tengan grandes problemas de salud" (Enfermera 5)

"No tenemos que luchar con toxicómanos... Una me ha venido. Pero que vamos, que no están enfermos" (Enfermera 7)

"Hace poco tiempo también tuve otro [...]. Que para nada ha menospreciado, ni ha echado por tierra el trabajo que yo realicé con él [...]. Todo lo contrario, como que lo respetan mucho. Me sorprende porque la idea que tenemos es diferente a lo que yo me encuentro luego en el trato con ellos" (Enfermera 1)

\section{Actitudes hacia la atención a la multicultu- ralidad}

La tendencia general muestra una actitud decidida a ofrecer cuidados al individuo sin distinción de nacionalidad, edad, sexo, ideología o religión. Esta perspectiva muestra el respeto hacia la libertad e igualdad como contempla el Código Deontológico enfermero:

"Yo mi trato pienso que son como los que vienen nuestros. Ni más ni menos. [...] Les exijo lo mismo" (Enfermera 7)

"Mi actitud es... yo intento tratarlos como a cualquier otra persona porque creo que además la mezcla de culturas es muy buena y enriquecedora" (Enfermera 3)

Esta postura se complementa con aquella que percibe la diversidad cultural como una fuente de riqueza para la profesión enfermera, situación novedosa que supone un reto adornado por aspectos tanto positivos -como son la adquisición de conocimientos y de experiencias que contribuyen al desarrollo de la Enfermería y al crecimiento personal- como negativos -derivados de las dificultades en los encuentros culturales que desembocan en sentimientos de frustración por la imposibilidad de practicar una asistencia de calidad-. Así lo narran algunas entrevistadas:

"Repercute positivamente porque es un reto para ti, es un reto tener una situación y darle solución a esa situación. Y también, eso positivamente, porque como profesional te motiva a formarte en algo distinto a lo que tú estás habituada. Y negativamente, por la frustración que tienes a lo mejor de no saber cómo puedes ayudar, o dónde tienes que formarte, o dónde conseguir la información. Y eso también cómo profesional te frustra. Entonces tiene las dos vertientes. Dependiendo de tu actitud hacia la pro- 
fesión, tu actitud hacia la vida, te puedes decantar por una cosa o por la otra" (Enfermera 2)

"Enriquece en cuanto a... la cultura, a la comida, por ejemplo, la forma de ifijate!... la forma simplemente de curar, de cuidar a un enfermo porque en un país sudamericano tienen unos medios, aquí hay otros. Entonces, hay cosas que no son tan malas de los otros países y se puede captar. $Y$ luego nosotros podemos enriquecerlos a ellos a lo mejor con más tecnología o más... $Y$ a lo mejor con menos humanidad, que ellos son más humanos" (Enfermera 6)

Son varios los informantes que, entendiendo la sociedad como un paisaje multicultural, muestran una actitud de apertura y empatía:

"[...] es importante abrir barreras $y$ que yo creo que el día de mañana seremos ciudadanos del mundo [...] deberíamos ya cambiar la mentalidad en ese aspecto. Porque nosotros somos los primeros con tal como está España [...] tenemos que ir fuera a otros países a ganarnos la vida” (Enfermera 1)

Pese a que algunas perspectivas sostienen contundentemente diversos prejuicios y estereotipos, en su gran mayoría se trata de una visión flexible que, pese a las generalizaciones, reconoce que existe variabilidad dentro de un mismo colectivo y conflictos en la relación con el usuario independientemente de su origen e idioma.

"[...] siempre hay alguna tanto española como extranjera que hace de sus consultas de aquella manera, pero eso de todos los países, de nosotras y las extranjeras" (Enfermera 8)

\section{Aspectos destacados del encuentro cultural en la atención de Enfermería}

Durante las entrevistas han emergido temas relacionados con las particularidades y dificultades que encuentran las enfermeras en su contacto con una población multicultural. Estos aspectos constituyen una barrera en el alcance de una relación terapéutica con el paciente, lo que genera sentimientos de frustración:

\section{a. Idioma}

El idioma es la principal barrera señalada y se asocia principalmente al colectivo asiático y al musulmán en menor medida. La comunicación es destacada como un elemento crucial en la relación con el paciente y también es un medio para comprender las pautas burocráticas y terapéuticas de nuestro sistema sanitario, como reflejan estos cortes de entrevistas:

"[... si no nos entendemos no hay comunicación. [...]Entonces sí que es verdad que sin un traductor y no entienden nada, pues es difícil que tú les puedas dar en las revisiones explicaciones. Les puedes hacer lo mínimo. [...] O sea que ellos te dicen: "Sí, sí, sí..."Pero ellos hacen lo que entienden. Lo que entienden que está bien, que para ellos está bien porque en su país así lo hacen" (Enfermera 8)

\section{b. Educación para la Salud}

La Educación para la Salud es una herramienta fundamental en la Enfermería Familiar y Comunitaria. La complejidad del nuevo contexto multicultural no solo radica en la barrera idiomática, sino en el choque cultural referido a las diferencias respecto a estilos de vida. Las entrevistas desvelan que los cuidados se ofrecen desde un enfoque etnocéntrico, es decir, en función de lo propio como lo adecuado y 
lo ajeno como inadmisible (Rico, 2009). No obstante, hay informantes que son conscientes del papel que juegan las diferencias culturales cuando se trata de alcanzar objetivos a través de intervenciones enfermeras relacionadas con el estilo de vida, con lo que buscan acoplar sus indicaciones a las particularidades del paciente. Por otro lado, algunos argumentos apuntan a que son muchos los extranjeros que han atravesado un proceso de enculturación y se encuentran "occidentalizados".

"No aceptan que están en un país que no es el suyo y que tenemos una forma diferente de ver las cosas, sino que implantan sus normas y toda su... pues todo lo que conlleva su cultura: su alimentación, todo lo que enfermería tiene que trabajar con ellos. Los programas de educación de... que entran, la alimentación, el ejercicio... [...] unas veces porque no te entienden y otras porque ellos no quieren dejar su cultura $y$ adaptarse a lo que tenemos aquí y a los consejos que tú les das" (Enfermera 4)

"[...]porque son hábitos y costumbres, son distintas, entonces es muy difícil llegar a ese usuario porque con lo que yo le explico a un usuario de mi misma cultura, mi misma tradición, mi misma religión... me puede entender. Pero en otra persona que todo eso sea distinto a lo mío, yo tengo que hacer un gran esfuerzo por intentar acoplar mis servicios a sus necesidades y a sus costumbres" (Enfermera 2)

"[...] no puedes cambiar los hábitos de una persona que tiene una cultura, que vive de esta manera, que come de esta manera y que tal, porque yo le diga el que lo tenga que hacer como lo hacemos nosotros: no. Tienes que buscar una forma de adaptar lo de ellos a lo nuestro... saltando la barrera del idioma" (Enfermera 1)
La gran mayoría de las informantes ha subrayado limitaciones asociadas a la impartición de Educación para la Salud dirigida a los hábitos alimenticios, lo que es habitual teniendo en cuenta que la dieta es uno de los pilares básicos del tratamiento de enfermedades crónicas y de la promoción de la salud. Principalmente, han destacado dificultades en el control de la Diabetes Mellitus en pacientes asiáticos, así como en el control del peso en el colectivo sudamericano y musulmán, derivadas del hecho de que su cultura alimentaria incorpora alimentos ricos en hidratos de carbono en gran proporción, según explica este grupo de enfermeras. En este punto es preciso atender asimismo al nivel socioeconómico y su relación con la adopción de estilos de vida saludables.

"Ahora tengo concretamente una paciente que es una chica joven, diabética $y$ de origen chino. Con lo que ella habla muy bien español... con que esa barrera ya la hemos salvado. [...] estudia bachiller, con un nivel cultural medio pero con unas costumbres de alimentación que no tienen nada que ver con las nuestras. [...] el endocrino le pone dieta de $1500 \mathrm{kcal} y$ claro imagínate cómo le voy... Mi problema es que yo no puedo ofrecerle una dieta de $1500 \mathrm{kcal}$ [menú prefijado basado en la dieta mediterránea] a una persona que su alimentación no tiene nada que ver con las nuestras" (Enfermera 2)

"Los latinoamericanos creo que tienen una cultura alimenticia muy distinta a la nuestra y que influye muchísimo sobre todo en los pacientes diabéticos que tenemos día a día en nuestra consulta. Yo me he visto aquí a un paciente diabético y cuando, bueno... te crees que desayunan a lo mejor pues lo que nosotros desayunamos... Y me ha 


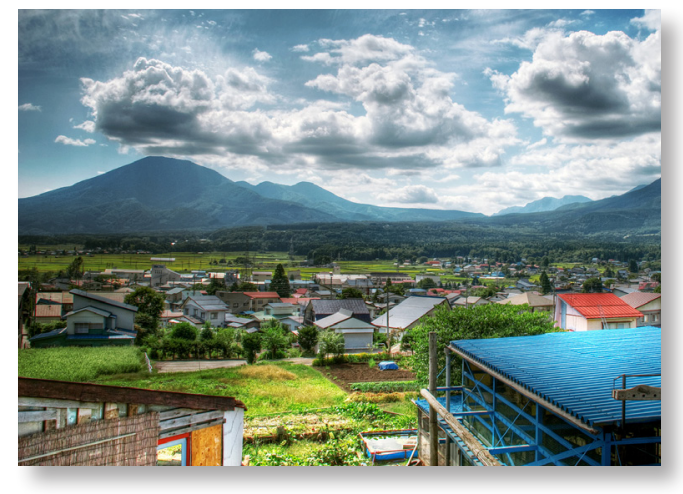

llamado la atención que ellos toman mucho arroz. Entonces, arroz en el desayuno... [... $]_{i} Q$ Qué hago yo? ¿Cómo los ayudo? Porque claro, la situación económica de ellos es muy distinta a lo que otros pacientes pueden permitirse. Entonces, incidir a lo mejor en la alimentación de unos pacientes con ese nivel adquisitivo, cuesta mucho" (Enfermera 3)

\section{Estrategias adoptadas en la práctica clínica}

La estrategia más extendida es la que trata de franquear la barrera lingüística, generalmente pidiendo al paciente que venga con un acompañante que hable castellano, aunque también se menciona la utilización del intérprete sanitario, así como recursos propios de la enfermera que mejoren la comunicación: habla más pausada, repetición, etc. En complemento, la empatía como instrumento fundamental en la práctica enfermera constituye otro de los elementos empleados. Solo una de las enfermeras puntualiza recurrir a la entrevista clínica en profundidad para valorar el estilo de vida de algunos pacientes.

"Entonces creo que son las estrategias más importantes: primero intentar empatizar con ellos $y \ldots$.. el idioma" (Enfermera 2)

"Es mucha educación sanitaria, intentar que te entiendan por encima de todo. Primero que la barrera idiomática sea la mínima. Utilizar las palabras que tú sabes que ellos van conociendo, ser muy repetitiva, repetir muchas veces lo mismo para que se les quede el concepto. $Y$ tienes que ir mucho más despacio. [...] A la hora de trabajar es un sobreesfuerzo. [...] si con una persona en una consulta, en una programada tú necesitas 15 minutos, con esa persona necesitas media hora cada vez que viene. Entonces te duplica el tiempo en todo" (Enfermera 3)

"Por ejemplo, un chino que sea diabético y no te entienda es muy difícil. Suelo decirle que venga con alguien que sepa español para explicarle» (Enfermera 6)

"En su cultura no profundizo más. A lo mejor, no por falta de interés sino porque tampoco veo tan importante estudiarme su cultura entera. Pero para llegar a aspectos concretos que son donde quiero yo llegar, que ahí es donde miro yo un poquito pero normalmente preguntándoles a ellos" (Enfermera 1)

\section{Formación y diversidad cultural}

Tras preguntar a estas enfermeras acerca de la posibilidad de recibir formación o incrementar sus conocimientos acerca de lo que ellas habían descrito, observamos que casi la totalidad apuntaba hacia la ausencia de recursos para abordar una circunstancia tan relevante y novedosa - por lo que son las propias enfermeras quienes salvan los impedimentos con recursos propios y en colaboración con otros profesionales-. Algunas posturas se mostraban abiertas a recibir cursos para mejorar la relación con el paciente inmigrado y para ampliar los recursos en su atención; por el contrario, otras no lo consideran tan conveniente, si bien una de las enfermeras señala que la adquisición de esos conocimientos sería 
una acción autodidacta. Así lo señalaban algunas de las enfermeras entrevistadas:

"Recursos propios porque no tenemos ninguna formación a pesar de los años $y$ a pesar de la diversidad cultural... no tenemos ninguna oferta en concreto de formación para la diversidad cultural. [...] actualmente en el sistema sanitario no hay un enfoque directo hacia ese problema. Ahí está y se va solventando como se va pudiendo" (Enfermera 2)

“Formación? Sí, pero yo pienso que esa formación puede ser más bien autodidacta. Quiero decir, que no es que se necesite mucha información, vamos, creo yo" (Enfermera 8)

\section{CONCLUSIÓN}

Este grupo de enfermeras describe la presencia de población multicultural como una realidad patente asociada principalmente a los movimientos migratorios transcurridos durante los últimos 10 años. En este sentido, relacionan la diversidad cultural con la figura del inmigrante económico que ya definíamos. Son escasos los discursos que se refieren al inmigrante comunitario frente al no comunitario, o a la llamada inmigración del bienestar (Rico, 2009). Estimamos que esta asociación diversidad cultural/inmigrante económico tiene implicaciones tanto cuantitativas -dado el incremento de los movimientos migratorios en la última década-, como cualitativas -las cuales están ligadas a la visibilidad selectiva que aporta el discurso mediático-. Esto último quiere decir que, de la cosmovisión que la población general tiene de la inmigración, sobresalen propiedades negativas alimentadas por los medios y de las que no siempre existe evidencia: la delincuencia, la drogadicción, el abuso de las prestaciones sociales, la conflicti- vidad, la enfermedad como patología importada del lugar de origen -frente a la patología de destino, cuya causa se halla en las dificultades del proceso migratorio-; así como lo exótico y lo mágico de la cultura del inmigrado frente a la realidad en su nuevo contexto. Es en este caldo de cultivo donde desarrollan las interpretaciones que originan un círculo vicioso de prejuicios y estereotipos.

Según los informantes y de acuerdo con Rico (2009) el idioma, la cultura y la religión son los principales elementos diferenciadores. Del análisis bibliográfico y del trabajo de campo extraemos que la principal barrera identificada por los profesionales sanitarios, y en concreto los enfermeros, es la lingüística (Llosada et al, 2010; Plaza, 2008; Moreno, 2008; Rico, 2012). Plaza (2008) apunta cómo este entorpecimiento en la comunicación constituye una dificultad en el acercamiento al paciente y en el establecimiento de la relación terapéutica de forma que, conforme a una ley de mínimos, no existe una mala praxis como tampoco se alcanza la excelencia en los cuidados. Frecuentemente, justificar la problemática de la relación enfermera-paciente inmigrado con la barrera lingüística opaca los déficits relacionados con la competencia cultural (Moreno, 2008).

Respecto a las estrategias que este grupo de enfermeras invoca para abordar el aspecto lingüístico, prevalece valerse de un acompañante del paciente que conozca el idioma, o la utilización de intérpretes sanitarios. No obstante, la bibliografía consultada destaca que la presencia de una tercera persona -acompañante y/o intérprete- es un elemento distorsionador en la comunicación (Cioffi, 2003). El abordaje de la barrera lingüística se acompaña para algunos de sobrecarga asistencial derivada de un aumento del tiempo de asistencia en la consulta, punto en el cual algunos autores proponen 
flexibilizar las agendas de las enfermeras (Rico, 2009).

En cuanto al papel que juega la cultura en la relación enfermera-paciente inmigrado, los discursos recogidos se proyectan hacia la problemática que entrañan la diferencia de estilos de vida en la impartición de educación sanitaria. En este sentido, la alimentación es el aspecto más destacado tanto por las enfermeras entrevistadas como por la bibliografía señalada. Las dificultades se acentúan cuando se trata de dar consejo dietético en el colectivo asiático, circunstancia en la cual se señala el déficit de recursos materiales cultural y lingüísticamente adaptados para esta clase de actividades asistenciales, o el desconocimiento que los profesionales pueden tener de la disponibilidad de los mismos si los hubiera. Igualmente, existen argumentos acerca del elevado consumo de hidratos de carbono, lo que deriva en dificultades en el control del peso y/o de la Diabetes Mellitus. Este fenómeno -como señalábamoses atribuible a diferencias culturales pero también al factor socioeconómico. Frecuentemente, estas diferencias no están vinculadas con la cultura de origen ni la de destino, sino más bien con las condiciones establecidas en esta: precariedad de las condiciones socio laborales con horarios de trabajo extensos y sueldos reducidos que favorecen la obesidad en niños y adultos (Moreno, 2008). La salud y el bienestar, en circunstancias adversas, pueden no ser una prioridad entre los objetivos de la familia como cabría esperar por parte de los profesionales sanitarios (Rico, 2009).

Más allá de los obstáculos identificados como potenciales y/o reales en la asistencia sanitaria al paciente inmigrado, de la percepción general recogida se extrae un conglomerado de prejuicios y estereotipos asociados a los colectivos que las entrevistadas describen. El estereotipo se caracteriza por ser "más simple que complejo o diferenciado, ser más falso que verdadero, haber sido adquirido de segunda mano más que por experiencia propia, y ser resistente al cambio" (Rico, 2009). En este sentido, Rico compara el estereotipo con la idea y la discriminación con la acción y añade: "El prejuicio guía la acción”. Sin embargo, cuando examinamos las actitudes que las enfermeras entrevistadas sostienen en la atención a la salud, encontramos actitudes positivas: búsqueda de la igualdad, miradas abiertas al cambio -la diversidad cultural como un reto-, al enriquecimiento que acompaña a la convivencia con diversas culturas, comprensión hacia la adversidad de sus circunstancias, empatía al rememorar a personas cercanas que emigraron y/o que están emigrando de manera forzada, reconocimiento de su función socio-laboral, etc.

Pese a relacionar la inmigración con aspectos problemáticos y generalizarlos, en la práctica diaria los tropiezos asociados a estos fenómenos -la conflictividad, la enfermedad importada, la drogadicción, etc.-, no forman parte de la realidad asistencial, a excepción de casos puntuales, como clarifica una de las enfermeras entrevistadas: "Me sorprende porque la idea que tenemos es diferente a lo que yo me encuentro luego en el trato con ellos" (Enfermera 1).

Otro de los aspectos a destacar es el enfoque etnocéntrico de los cuidados: en su mayoría, las enfermeras entrevistadas buscan la adaptación por parte del inmigrante a "lo nuestro". En este sentido, solo una pequeña porción de las enfermeras entrevistadas, manifiesta tener autoconciencia de las diferencias culturales, del enfoque etnocéntrico de los cuidados, de los propios prejuicios y del papel que juega la cultura en la percepción de la salud. 
Se encuentran en un estado de incertidumbre en tanto que no saben cómo abordar cuestiones relacionadas con la comunicación y la distancia cultural; y reclaman formación cuando se les pregunta acerca de ello. En otros casos no existe esa conciencia cultural, de la que se habla en términos de competencia cultural, y relacionan la oferta de formación con una compilación de características de la cultura del país de origen -enfoque a través del cual se descontextualizaría al paciente-, más que con un abordaje ajustado a la circunstancia real del proceso migratorio. En cualquier caso, el sumatorio de los resultados reafirma la necesidad de una intervención formativa en este aspecto.

De acuerdo con la bibliografía consultada y conforme a las interpretaciones extraídas de nuestros resultados, existe un problema de base en la comunicación con el paciente. La intervención en el manejo de esta realidad, más allá de las carencias expuestas, iría enfocada -citando a Rico (2009) - a aumentar la sensibilidad étnica o cultural entendida como "la actitud constructiva hacia las diferencias culturales". En este punto, se proporcionaría una atención culturalmente apropiada a través de "los conocimientos necesarios para procurar la mejor atención de salud a pacientes de diferentes culturas" y culturalmente competente, en tanto que la enfermera "entiende y atiende al contexto de la situación del paciente, circunstancias de emigración, factores de estrés y diferencias culturales". Damos por hecho que la disponibilidad de material cultural y lingüísticamente adaptado, debería ser un mínimo en la asistencia a población multicultural.

Finalmente, y respecto a posibles investigaciones futuras, consideramos necesario contrastar los discursos de las enfermeras con la voz de los pacientes inmigrantes, de modo que podría ser elaborado un estudio con una perspectiva más holística. Existen escasas investigaciones que realicen un amplio trabajo de campo desde una perspectiva antropológica -entrevistas en profundidad, grupos de discusión, observación participante, etc.-, por lo que proponemos ampliar el número de estudios tanto en Atención Primaria como Atención Especializada. No obstante, sería interesante desarrollar una intervención formativa sobre la base de los estudios ya realizados con el fin de aportar a las enfermeras una visión más ajustada a la realidad de la población inmigrante, así como para generar un aumento de la conciencia y sensibilidad cultural.

\section{BIBLIOGRAFÍA:}

- Belintxon, M., y López-Dicastillo, O. (2010) Los retos de la promoción de la salud en una sociedad multicultural: revisión narrativa de la literatura. Anales Sistema Sanitario Navarra, 37(3), 401-409. doi: 10.4321/S113766272014000300009

- Burns, N., y Grove, S. (2012). Investigación en enfermería: Desarrollo de la práctica enfermera basa en la evidencia. Barcelona: Elsevier Saunders.

- Cioffi, R.N. (2003). Communicating with culturally and linguistically diverse patients in an acute care setting: nurses' experiencies. International Journal of Nursing Studies, 40(3), 299-306. doi: 10.1016/S00207489(02)00089-5

- Consejería de Economía y Hacienda. (2012). Plan Estratégico de la Región de Murcia (2014-2020): Diagnóstico general de la situación económica (8-9). Recuperado de http://planiris2020.carm.es/web/pagina?IDCONTENI $\underline{\mathrm{DO}}=47453 \& \mathrm{IDTIPO}=100 \& \mathrm{RASTRO}=\mathrm{c} \$ \mathrm{~m} 47432$

- Dept. of Ethics, Trade, Human Rights and Health Law (OMS). (2005). Migración internacional, salud y derechos humanos. Ginebra: OMS (7). Recuperado de http://www. who.int/hhr/activities/2005\%20PRT\%2016325\%20 ADD\%201\%20Migr_HHR-Spanish\%20edition.pdf 
- Fuertes, M.C., de Andrés, M.R., García-Castellano, P., Urmeneta, S., Uribe, J.M., y Bustince, P. (2010). Atención al paciente inmigrante: realidad y percepción de los profesionales de 6 zonas de Atención Primaria de Navarra. Anales Sistema Sanitario Navarra, 33(2), 179-190. Recuperado de: http://scielo.isciii.es/scielo.php?pid=S11 3766272010000300006\&script=sci_arttext

- Gentil, I. (2010). Competencia cultural en Enfermería: Población Subsahariana. Cultura de los cuidados, 27(1), 61-67. Recuperado de http://rua.ua.es/dspace/bitstream/10045/14391/1/CC_27_08.pdf

- Instituto Nacional de Estadística (INE). (2014). Cifras de población a 1 de Enero de 2014. Estadística de migraciones 2013. Datos provisionales. Madrid: INE. Recuperado de http://www.ine.es/prensa/np854.pdf

- Jociles, M.I. (1999). Las técnicas de investigación en antropología. Mirada antropológica y proceso etnográfico. Gazeta de Antropología, 15(1). Recuperado de: http:// www.ugr.es/ pwlac/G15 01MariaIsabel Jociles Rubio. $\underline{\mathrm{html}}$

- Leno, D. (2006). Buscando un modelo de cuidados de enfermería para un entorno multicultural. Gazeta de Antropología, 22(32). Recuperado de http://www.gazetaantropologia.es $/ \mathrm{p}=2681$

- Lillo, M. y Casabona, I. (2006). Fenómenos migratorios, competencia cultural y cuidados de salud. Cultura de los cuidados, 20(2), 87-91. Recuperado de:http://culturacuidados.ua.es/enfermeria/article/view/250/499
- Llosada, J., Vallverdú, I., Miró, M., Pijem, C., y Guarga, A. (2010). Acceso y uso de los servicios sanitarios por parte de los pacientes inmigrantes: la voz de los profesionales. Atención Primaria, 44(2), 82-88. doi: 10.1016/j. aprim.2010.11.014

- Ministerio de Trabajo e Inmigración. (2011). Plan Estratégico de Ciudadanía e Integración (2011-2014). Madrid: Dirección General de Integración de los Inmigrantes. Recuperado de http://extranjeros.empleo.gob.es/es/Programas_Integracion/ Plan_estrategico2011/pdf/PECI-2011-2014.pdf

- Moreno, M. (2008). El cuidado del "otro": Un estudio sobre la relación enfermera/paciente inmigrado. Barcelona: Edicions Bellaterra.

- Plaza, F.J. (2008). Competencia Comunicativa Intercultural de los profesionales de Enfermería con pacientes inmigrantes musulmanes. (Tesis doctoral, Universidad de Almería).Recuperado de http://migraciones.ugr.es/ cddi/images/tesis/PlazaDelPino2008.pdf

- Perdiguero, E., y Comelles, J.M. (2000). Medicina y cultura: Estudios entre la antropología y la medicina. Barcelona: Edicions Bellaterra.

- Rico, J.I. (2009). El inmigrante "enfermo". Murcia: Ediciones Isabor.

- Serra, M., Mestres, L., González, M., Leyva, J.M., de Dios, R. y Montiel, M. (2013). Competencia clínico cultural: Análisis de la capacitación de los profesionales de la salud. Index Enfermería, 22(1-2),16-19. doi: 10.4321/ S1132-12962013000100004 